\title{
Indolent evolution of an episode of acute gastroenteritis complicated with portal pneumatosis
}

\author{
A. Mancebo ${ }^{1}$, E. Álvarez-Hornia ${ }^{2}$, M. Rodríguez-Peláez ${ }^{1}$, M. Ibáñez ${ }^{1}$, L. H. Luyando² and M. Varela ${ }^{1}$ \\ ${ }^{\prime}$ Department of Gastroenterology and Hepatology. ${ }^{2}$ Department of Radiology. Hospital Universitario Central de Asturias. \\ Oviedo. Asturias, Spain
}

\begin{abstract}
The discovery of gas in the portal system related to indolent clinical settings has increased in recent years due to a growth in the performance of imaging tests. We report the first case of spontaneous resolution of portal pneumatosis due to acute gastroenteritis described in the literature.
\end{abstract}

Key words: Portal pneumatosis. Acute gastroenteritis. Streptococus viridans.

\begin{abstract}
RESUMEN
El hallazgo de gas en el sistema portal en relación a cuadros de evolución indolente ha aumentado en los últimos años por la mayor realización de pruebas de imagen. Creemos importante destacar que, a pesar de los hallazgos radiológicos, el sentido clínico es lo más importante a la hora de enfocar el diagnóstico. Presentamos el primer caso en la literatura de neumatosis portal secundaria a un cuadro de gastroenteritis aguda, con resolución espontá-
\end{abstract} nea.

Palabras clave: Neumatosis portal. Gastroenteritis aguda. Streptococus viridans.

Mancebo A, Álvarez-Hornia E, Rodríguez-Peláez M, Ibáñez M, Luyando LH, Varela M. Resolución espontánea de neumatosis portal tras un episodio de gastroenteritis aguda. Rev Esp Enferm Dig 2010; 102: 557-559.

\section{INTRODUCTION}

Portal pneumatosis is a relatively uncommon radiological finding in the clinical practice. In most cases this entity is associated with an acute abdominal disease and a high mortality rate. In the emergency it is necessary to rule out the presence of necrosis or ischemic bowel, which may require an urgent laparotomy.

Here we present a patient with portal pneumatosis secondary to an episode of acute gastroenteritis solved with conservative management.

Received: $15-03-10$

Accepted: 26-03-10.

Correspondence: María Varela. Sección de Hepatología. Servicio de Aparato Digestivo. Hospital Universitario Central de Asturias. C/ Celestino Villamil, s/n.33006 Oviedo, Asturias. e-mail: maria.varela.calvo@gmail.com

\section{CASE REPORT}

A male, 58 years old, entered the emergency department by cramping abdominal pain, vomiting, nausea and diarrhea of 2 days. His son had similar symptoms and both of them had eaten cooked quail two days earlier. The patient had prior diagnoses of benign prostatic hypertrophy and arterial hypertension, under treatment with angiotensin-II receptor antagonists and torasemide, as well as an episode of acute abdominal pain secondary to duodenitis after taking ibuprofen, 6 months before, which had been solved with omeprazole. At admission the patient's basic physical examination, temperature, arterial tension and heart rate were normal. Blood count, renal function, serum amylase, lipase and liver function tests were within normal ranges, except serum bilirubin value of $1.80 \mathrm{mg} / \mathrm{dL}$ (upper normal limit $1.2 \mathrm{mg} / \mathrm{dL}$ ) and LDH (lactate dehydrogenase) of $547 \mathrm{U} / \mathrm{L}$ (upper limit of normal 460 $\mathrm{U} / \mathrm{L}$ ). The abdominal pain was so intense that the med- 
ical doctors of the emergency decided to perform an abdominal ultrasound. This showed small mobile hyperechoic foci within the main branch of the portal vein, with multiple hypoechoic areas distributed in both lobes suggestive of gas (Fig. 1). The gallbladder, the head of the pancreas and small bowel loops showed no abnormalities and there was no ascites. The only possible cause of the pneumatosis could be the presence of a thickening of the stomach wall, in possible connection with an emphysematous gastroduodenitis. We performed an abdominal-pelvic computed tomography (CT), which confirmed the presence of gas in the main portal vein and its distal branches and in both lobes (Fig. 2). Mesenteric ischemia inflammation of the gall bladder and pelvic disorders were ruled out. By contrast with the previous ultrasound test, the stomach wall appeared normal, although there was a minimum amount of gas in the short gastric vessels.

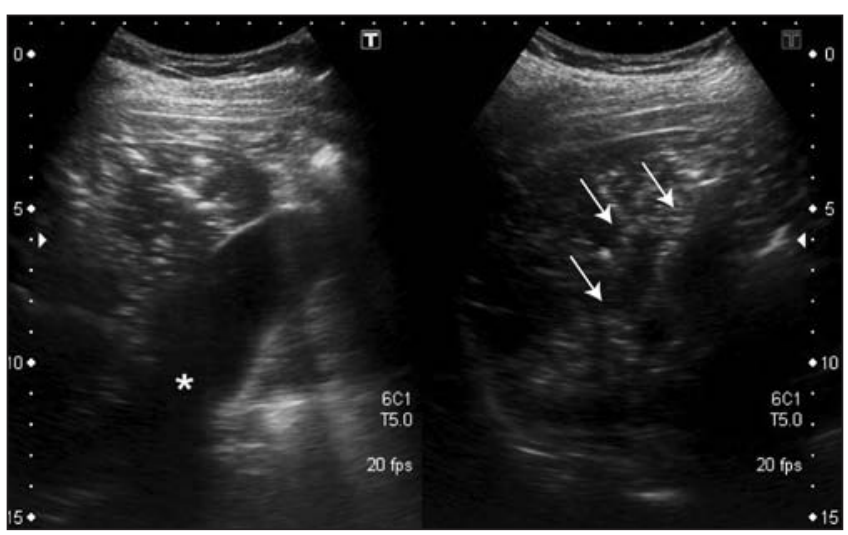

Fig. 1. Ultrasound imaging revealed hyperechoic foci moving inside the main portal vein, together with multiple spots and stripes distributed in both lobes of the liver, corresponding with gas (arrow signaling gas in right hepatic lobe). Gallbladder showed no abnormalities (*).

It seemed to be an uneventful mild acute case of gastroenteritis due to contaminated food. However, the patient was admitted to the hospital because of the portal pneumatosis. He received treatment with amoxicillinclavulamic together with low molecular weight heparin subcutaneously by the risk of pylephlebitis following the portal vein thrombosis. Stool cultures were negative, and the presence of Entamoeba histolytica, eggs and parasites were ruled out. In one blood culture grew up Streptococcus viridans. Four days later, the CT scan was repeated, and it depicted the disappearance of the portal pneumatosis. The patient was discharged completely recovered. Four months later he continued asymptomatic, a new ultrasound demonstrated total patency of the main portal trunk and subcutaneous heparin therapy was stopped.

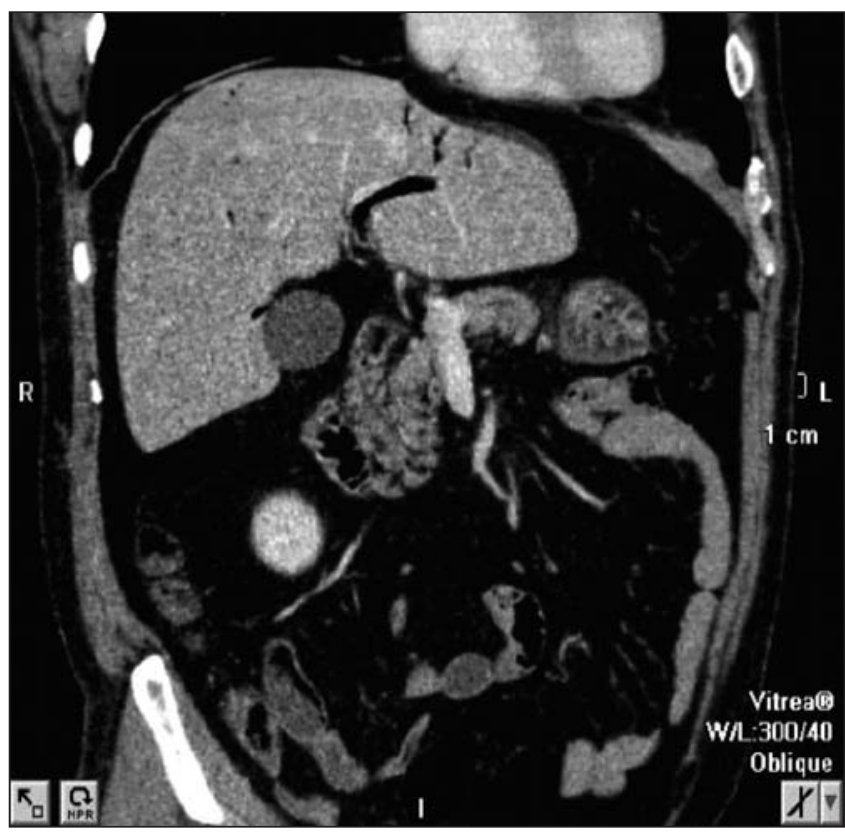

Fig. 2. The CT scan performed at admission revealed the presence of gas in the main portal vein and its branches.

\section{DISCUSSION}

The presence of portal pneumatosis is a relatively uncommon radiological sign, first described in 1955 in relation to necrotizing enterocolitis in newborns and traditionally linked to mesenteric necrosis in adults (1).

In a half of situations the coexistence of gas in the intestinal wall has been reported. The finding of portal pneumatosis together with the presence of necrosis or bowel ischemia has a mortality rate higher than $75 \%$ (2), and for that reason it requires an aggressive management, including surgical exploration.

However, several studies by Liebman (2) and Kinoshita (3) showed that if mucosal disruption, distended loops, abscesses or gastric ulcers are present, mortality figures do not exceed 20-30\% and the most appropriate management is probably an intensive observation. Therefore, the radiological detection of portal pneumatosis should not determine any clinical or surgical management per se.

The increased frequency of imaging tests, particularly $\mathrm{CT}$, has the detection of portal pneumatosis in contexts with less morbidity, such as diverticulitis, suppurative cholangitis, intestinal obstruction, inflammatory bowel disease or after endoscopic procedures. This radiographic finding may persist for a few minutes to several weeks.

In our case, the patient had consulted by an acute gastroenteritis probably secondary to ingestion of contaminated food, and due to the presence of cramping abdominal pain an ultrasound was performed. Despite an intensive search for a primary cause of ischemia or abdominal infection, no significant abnormalities on physical examination, lab exams or imaging tests were established. 
One of two blood cultures was positive for Streptococcus viridans. This gram-positive is not a gas producer and it belongs to the normal commensal flora of the oral, respiratory and gastrointestinal lumen (4). For those reasons it may be considered a contaminant. A blood culture can be positive for $\mathrm{S}$ viridans in case of trauma, dental manipulations, mucositis, upper respiratory tract surgery, instrumentation of the genitourinary or intestinal tract, etc. Our patient had not undergone any manipulation to justify such a bacteremia.

To our knowledge, this is the first case of extensive portal pneumatosis secondary to acute gastroenteritis with the presence of gas in the short vessels of the gastric camera. The clinical and radiological outcome was satisfactory.

\section{REFERENCES}

1. See C, Elliott D. Images in clinical medicine: pneumatosis intestinalis and portal venous gas. N Engl J Med 2004; 350(4): e3.

2. Liebman PR, Patten MT, Manny J, Benfield JR, Hechtman HB. Hepatic-portal venous gas in adults: etiology, pathophysiology and clinical significance. Ann Surg 1978; 187(3): 281-7.

3. Kinoshita H, Shinozaki M, Tanimura H, Umemoto Y, Sakaguchi S, Takifuji K, et al. Clinical features and management of hepatic portal venous gas: four case reports and cumulative review of the literature. Arch Surg 2001; 136(12): 1410-4.

4. Weinstein MP, Towns ML, Quartey SM, Mirrett S, Reimer LG, Parmigiani $\mathrm{G}$, et al. The clinical significance of positive blood cultures in the 1990s: a prospective comprehensive evaluation of the microbiology, epidemiology, and outcome of bacteremia and fungemia in adults. Clin Infect Dis 1997; 24: 584-602. 